\title{
Characteristics of gastric cancer in negative test of serum anti-Helicobacter pylori antibody and pepsinogen test: a multicenter study
}

\author{
Mariko Kiso $^{1} \cdot$ Masaharu Yoshihara $^{2}$ Masanori Ito $^{1} \cdot$ Kazuhiko Inoue $^{3}$. \\ Katsuaki Kato $^{4} \cdot$ Shigemi Nakajima $^{5} \cdot$ Katsuhiro Mabe $^{6} \cdot$ Masao Kobayashi $^{7}$. \\ Naomi Uemura ${ }^{8}$. Tomoyuki Yada ${ }^{8}$ - Masashi Oka ${ }^{9}$ Takashi Kawai ${ }^{10}$. \\ Tomoyuki Boda $^{11}$ - Takahiro Kotachi ${ }^{1} \cdot$ Kazuhiko Masuda $^{1} \cdot$ Shinji Tanaka $^{11}$. \\ Kazuaki Chayama ${ }^{1}$
}

Received: 30 September 2016/ Accepted: 8 December 2016/Published online: 26 December 2016

(C) The International Gastric Cancer Association and The Japanese Gastric Cancer Association 2016

\begin{abstract}
Background and aim The serological risk prediction system combines the pepsinogen test and anti-Helicobacter pylori (H. pylori) antibody determination. In this system, chronic atrophic gastritis (CAG) is diagnosed using the pepsinogen test. Patients who are $H$. pylori negative and pepsinogen negative are classified into group $\mathrm{A}$, are assumed to be $H$. pylori uninfected, and are at an extremely low risk for gastric cancer. However, gastric cancers are
\end{abstract}

Masaharu Yoshihara

myoshih@hiroshima-u.ac.jp

Mariko Kiso

kiso927@hiroshima-u.ac.jp

Masanori Ito

maito@hiroshima-u.ac.jp

Kazuhiko Inoue

inoueki@junpukai.or.jp

Katsuaki Kato

kkato@cat-v.ne.jp

Shigemi Nakajima

shigemin@ rainbow.plala.or.jp

Katsuhiro Mabe

katsumabe@me.com

Masao Kobayashi

kobayashim@kyoto2.jrc.or.jp

Naomi Uemura

nuemura@hospk.ncgm.go.jp

Tomoyuki Yada

tomoyuki0618@yahoo.co.jp

Masashi Oka

oka@dd.iij4u.or.jp

Takashi Kawai

t-kawai@tokyo-med.ac.jp detected in this group. The aim of this study is to clarify the clinicopathological status of group A patients with gastric cancer.

Methods A total of 109 gastric cancer patients classified as group A were enrolled in a multicenter study. Group A patients were divided into two subgroups: group AN ( $H$. pylori uninfected) and group AP (H. pylori infected). They were compared to $183 \mathrm{H}$. pylori-infected gastric cancer patients who were not in group A.

Tomoyuki Boda

t-bow5533@hiroshima-u.ac.jp

Takahiro Kotachi

kotachi@hiroshima-u.ac.jp

Kazuhiko Masuda

masu6743@hiroshima-u.ac.jp

Shinji Tanaka

colon@hiroshima-u.ac.jp

Kazuaki Chayama

chayama@hiroshima-u.ac.jp

1 Department of Gastroenterology and Metabolism, Hiroshima University, Hiroshima, Japan

2 Health Service Center, Hiroshima University, Higashi-Hiroshima, Japan

3 Department of General Medicine, Kawasaki Medical School, Kurashiki, Japan

4 Department of Cancer Detection Center, Miyagi Cancer Society, Sendai, Japan

5 Department of General Medicine, Gastroenterology and Health-care, Japan Community Health-care Organization Shiga Hospital, Otsu, Japan

6 Department of Gastroenterology, National Hospital Organization Hakodate Hospital/Cancer Preventive Medicine, Hokkaido University, Sapporo, Japan 
Results Of the 109 patients, only 7 were classified as group AN; the other 102 were classified as group AP. The clinicopathological features of group AP included older age, predominantly differentiated type cancer, endoscopically visualized $\mathrm{CAG}$, and pepsinogen $(\mathrm{PG}) \mathrm{I} / \mathrm{II}$ ratio lower than that of group AN. In group AN, the depressed type was dominant, and the PG I/II ratio was higher than in those gastric cancer patients who were infected with $H$. pylori. Conclusion Patients in group AP had CAG, and their gastric cancers were similar to those of $H$. pylori-eradicated patients. Concerning the recent $\mathrm{ABC}$ classification system, advanced decision criteria should be proposed to decrease the false-negative evaluation of gastric cancer risk.

Keywords Helicobacter pylori - Gastric cancer . Serological risk prediction system · Pepsinogen · Gastritis

\section{Introduction}

In Japan, gastric cancer is the second most common cause of cancer-related death [1]. It is well known that Helicobacter pylori (H. pylori) infection is a major risk factor for gastric cancer. In addition, the gastric mucosal inflammation and atrophy induced by $H$. pylori are important risk factors for gastric cancer [2-4].

As biomarkers for gastritis and corpus atrophy, serum pepsinogen (PG) levels are the most reliable and established markers for gastritis including corpus atrophy [5, 6]. PG concentrations have been used with a set of recommended cutoff values to diagnose chronic atrophic gastritis (CAG) (PG I $\leq 70 \mathrm{ng} / \mathrm{ml}$ and $\mathrm{PG} \mathrm{I} / \mathrm{II} \leq 3.0 \mathrm{ng} / \mathrm{ml}$ ) [7]. Therefore, gastric cancer risk can be evaluated by measuring the serum anti-H. pylori antibody titer in combination with PG levels [8-10], and this system of risk prediction using the anti- $H$. pylori antibody serological test and the PG test is known as the ABC classification system in Japan [8-10]. Subjects with negative test results for either the anti-H. pylori antibody serology test or the PG test were classified in group $\mathrm{A}$, anti- $\mathrm{H}$. pylori antibodypositive/PG-negative subjects were in group $\mathrm{B}$, anti- $H$.

7 Department of Health Care Division, Kyoto Second Red Cross Hospital, Kyoto, Japan

8 Department of Gastroenterology, Kohnodai Hospital, National Center for Global Health and Medicine, Ichikawa, Japan

9 Department of Gastroenterology and Hepatology, Faculty of Medicine, Saitama Medical University, Kawagoe, Japan

10 Department of Endoscopy Center, Tokyo Medical University Hospital, Tokyo, Japan

11 Department of Endoscopy, Hiroshima University Hospital, Hiroshima, Japan pylori antibody-positive/PG-positive subjects in group C, and anti-H. pylori antibody-negative/PG-positive subjects were in group D. Specifically, multivariate meta-analyses suggested that group A had a lower risk than groups B/C/D and that group B had a lower risk compared with groups C/D [10].

Indeed, the $\mathrm{ABC}$ classification system is now one of the efficient risk prediction tools utilized for gastric cancer screening. Because the development of gastric cancer in those patients not infected with $H$. pylori is extremely rare in Japan [11], it may be expected that the H. pylori-uninfected healthy population could be excluded from the mass screening system for gastric cancer. Group A includes subjects with neither $H$. pylori infection nor CAG by testing; however, group A contains $H$. pylori-infected subjects. Boda et al. reported that approximately $10 \%$ of patients with gastric tumors (cancer or adenoma) belong to group A [12]. The cancer risk of these patients may be underappreciated using the current criteria of the $\mathrm{ABC}$ classification system. The presence of $H$. pylori-infected subjects in group A is a crucial problem because the subjects in group A are judged as having an extremely low risk for gastric cancer, similar to healthy, $H$. pylori-uninfected individuals.

In the present study, we constructed a multicenter study to clarify the clinicopathological features of the gastric cancer patients in group A (having test results negative for serum anti-H. pylori antibody and PG), and we discuss and advocate for the advancement of methods for evaluating gastric cancer risk using serum markers.

\section{Methods}

\section{Patients}

A total of 109 patients [ 82 men and 27 women of mean age 68.8 years (range, 40-87 years)] with 114 lesions were registered in this study from seven collaborating research units within hospitals (Hiroshima University Hospital, Kawasaki Medical School, Center of Cancer Screening Miyagi Cancer Society, Hokkaido University, Social Insurance Shiga Hospital, Kyoto Second Red Cross Hospital, Kohnodai Hospital). These subjects were patients with gastric cancer who underwent gastrointestinal endoscopic examinations, measurement of anti-H. pylori antibody titers (Eiken E-plate test), and serum PG I and II levels (CLIA Abbott; E-plate Eiken or LZ test Eiken) between January 2003 and October 2014. The diagnosis of gastric cancer was based on the following: $\mathrm{ABC}$ methods (1), X-ray examination (14), gastrointestinal endoscopy (11), visiting clinics with symptoms (4), and unknown (79). Five patients had received $H$. pylori eradication therapy, 
and proton pump inhibitors (PPI) were administered to 12 patients.

For the analysis, we classified group A patients into two subgroups: $H$. pylori uninfected and $H$. pylori infected. We defined the $H$. pylori-uninfected group A patients as those showing negative results in other $H$. pylori examinations [histological examination, urea breath test (Otsuka, Tokushima, Japan), rapid urease test, anti-H. pylori antibody in the urine, stool antigen test, or microbial culture test] and having no atrophic gastritis. The $H$. pylori-infected group A patients were defined as either those showing positive results in the $H$. pylori test or those having CAG. Moreover, it is speculated that the $H$. pyloriinfected group A consists of $H$. pylori-infected and formerly infected subjects. In this study, the H. pylori-uninfected group A gastric cancer patients are shown as "group AN," meaning group A with negative results for $H$. pylori infection tests. The $H$. pylori-infected group A is shown as "group AP," meaning group A patients who are positive for $H$. pylori infection.

\section{Group BCD gastric cancer patients}

To clarify the clinicopathological characteristics of gastric cancer in group A, we enrolled gastric cancer patients who were classified into group B, C, or D between June 2003 and October 2014 in Hiroshima University Hospital ( $n=167)$, Shiga Hospital $(n=6)$, or Kawasaki Medical hospital $(n=10)$. Regarding the patients in Hiroshima University Hospital, we chose the patients from the database used in the previous study by Boda et al. [12]. These 183 gastric cancer patients (136 men; mean age, 66.4 years) with $H$. pylori-positive results or with CAG were used as controls. We excluded those patients with gastric adenoma, those who were post gastrectomy, and those who had severe renal dysfunction. The use of PPI was checked during a medical interview. Fasting sera were similarly collected for the measurement of anti- $H$. pylori antibody titers (Eiken E-plate test) and serum PG I and II levels (CLIA Abbott; E-plate Eiken, LZ test Eiken).

\section{Measurement}

We used the $\mathrm{ABC}$ classification system as described previously $[8,9]$. Fasting sera were obtained from all patients, and serum PG I and II levels (CLIA Abbott, E-plate Eiken; LZ test Eiken, Tokyo) and anti-H. pylori antibody titers (Eplate) were evaluated. In the PG test, a set of cutoff values (PG I $\leq 70 \mathrm{ng} / \mathrm{ml}$ and PG I/II $\leq 3.0 \mathrm{ng} / \mathrm{ml}$ ) was used for the diagnosis of atrophic gastritis. If PG levels were less than the cutoff values, they were considered as positive for the test. In the anti-H. pylori antibody titer test, the cutoff value for the diagnosis of $H$. pylori infection was more than
$10.0 \mathrm{U} / \mathrm{ml}$. Because the lower sensitivity limit is $3.0 \mathrm{U} / \mathrm{ml}$ in this kit, antibody titers between 3 and $9.9 \mathrm{U} / \mathrm{ml}$ are considered within the measurable range of a negative test result ("negative-high test").

\section{Endoscopic findings}

The endoscopic grade of gastric mucosal atrophy was evaluated on the basis of the endoscopic classification system described by Kimura and Takemoto [13], which was used to classify cases into seven grades $(\mathrm{C} 0, \mathrm{C} 1, \mathrm{C} 2$, $\mathrm{C} 3, \mathrm{O} 1, \mathrm{O} 2, \mathrm{O} 3)$. Information concerning the gastric cancer (size, endoscopic gross type, histological type, location, and depth of invasion) was recorded according to the Japanese Classification of Gastric Carcinoma [14]. For comparison, the histological type was divided into two groups in this report: differentiated type (tubular adenocarcinoma and papillary adenocarcinoma) and undifferentiated type (poorly differentiated adenocarcinoma and signet-ring cell carcinoma).

\section{Statistical analysis}

The statistical analysis was performed using the chi-square test and analysis of variance (ANOVA). For multiple comparisons, the Steel-Dwass method was used. When $p<0.05$, differences were recognized as statistically significant. Statistical analysis was performed using JMP (SAS Institute).

\section{Ethics}

The protocol was approved by the ethics committee of Hiroshima University Hospital (no. Epd-1029-1).

\section{Results}

\section{Clinicopathological characteristics in each group}

Clinical and pathological data are summarized in Table 1. Among 109 patients in group A, only 7 (6.4\%) were included in the $H$. pylori-uninfected group A (group AN). The H. pylori-infected group A (group AP) consisted of $102(93.6 \%)$ of 109 patients, and all these patients had CAG that was visible endoscopically. In Table $1, p$ values are shown for comparisons between group $\mathrm{AN}$ and group $\mathrm{AP}$, between group $\mathrm{AN}$ and group $\mathrm{BCD}$, and between group AP and group $\mathrm{BCD}$, respectively.

There was a male dominance in all groups. In group $\mathrm{AN}$, the median age of the patients was younger than that in group AP $(p=0.018)$ and group $\operatorname{BCD}(p=0.086)$. Group AP was slightly older than group BCD 
Table 1 Clinicopathological characteristics of patients

\begin{tabular}{|c|c|c|c|c|c|c|}
\hline & Group AN & $p$ value vs. AP & $p$ value vs. BCD & Group AP & $p$ value vs. $\mathrm{BCD}$ & Group BCD \\
\hline $\operatorname{Sex}(M / F)$ & $5 / 2(7)$ & 0.810 & 0.87 & $77 / 25(102)$ & 0.83 & $136 / 47(183)$ \\
\hline Age (mean age $\pm \mathrm{SD}$, median) & $\begin{array}{l}57.3 \pm 10.87 \\
61\end{array}$ & 0.018 & 0.086 & $\begin{array}{l}69.5 \pm 9.10 \\
70\end{array}$ & 0.077 & $66.4 \pm 9.89,67$ \\
\hline Medical history & & 0.570 & 0.018 & & 0.016 & \\
\hline Post $H$. pylori eradication & 0 & & & 5 & & 6 \\
\hline PPI using & 2 & & & 10 & & 0 \\
\hline Operation of stomach & 0 & & & 1 & & 0 \\
\hline $\begin{array}{l}\text { Tumor size } \\
(\mathrm{mm}, \text { mean size } \pm \mathrm{SD}, \text { median })\end{array}$ & $10.4 \pm 6.76,9$ & 0.048 & 0.0068 & $\begin{array}{c}16.0 \\
15\end{array}$ & 0.008 & $\begin{array}{l}21.2 \pm 14.32 \\
20\end{array}$ \\
\hline Macroscopic type $^{\mathrm{a}}$ & $n=7$ & 0.8041 & 0.65 & $n=107$ & 0.21 & $n=201$ \\
\hline 0-I (protruding) & 0 & & & $2(1.87 \%)$ & & $5(2.49 \%)$ \\
\hline 0-IIa (superficial elevated) & 0 & & & $22(20.6 \%)$ & & $65(32.3 \%)$ \\
\hline 0-IIb (superficial flat) & 0 & & & 0 & & $3(1.49 \%)$ \\
\hline 0-IIc (superficial depressed) & $7(100 \%)$ & & & $80(74.8 \%)$ & & $125(62.2 \%)$ \\
\hline Type 1 (mass) & 0 & & & $1(0.93 \%)$ & & $1(0.50)$ \\
\hline Type 2 (ulcerative) & 0 & & & $1(0.93 \%)$ & & 0 \\
\hline Type 3 (infiltrative ulcerative) & 0 & & & 0 & & 0 \\
\hline Type 4 (diffuse infiltrative) & 0 & & & $1(0.93 \%)$ & & $1(0.50)$ \\
\hline Type 5 (unclassifiable) & 0 & & & 0 & & $1(0.50)$ \\
\hline Histological type & $n=7$ & $<0.0001$ & $<0.0001$ & $n=107$ & 0.87 & $n=201$ \\
\hline Differentiated & 0 & & & $93(86.92 \%)$ & & $176(87.56 \%)$ \\
\hline Undifferentiated & $7(100 \%)$ & & & $14(13.08 \%)$ & & $25(12.44 \%)$ \\
\hline Location & $n=7$ & 0.31 & 0.55 & $n=107$ & 0.43 & $n=201$ \\
\hline Upper third & 0 & & & $15(14.02 \%)$ & & $22(10.95 \%)$ \\
\hline Middle third & $1(14.29 \%)$ & & & $38(35.51 \%)$ & & $59(29.35 \%)$ \\
\hline Lower third & $6(85.71 \%)$ & & & $53(21.50 \%)$ & & $119(59.20 \%)$ \\
\hline Unclassifiable & 0 & & & $1(0.93 \%)$ & & $1(0.50 \%)$ \\
\hline Depth of invasion & $n=7$ & 0.99 & 1.00 & $n=107$ & 0.48 & $n=201$ \\
\hline M (mucosa) & $6(85.71 \%)$ & & & $82(77.36 \%)$ & & $169(84.08 \%)$ \\
\hline SM (submucosa) & $1(14.29 \%)$ & & & $21(19.81 \%)$ & & $29(14.43 \%)$ \\
\hline MP (muscularis propria) & 0 & & & 0 & & 0 \\
\hline SS (subserosa) & 0 & & & $1(0.94 \%)$ & & $1(0.50 \%)$ \\
\hline SE (serosa) & 0 & & & 0 & & $1(0.50 \%)$ \\
\hline $\begin{array}{l}\text { SI (invades adjacent } \\
\text { structures) }\end{array}$ & 0 & & & $1(0.94 \%)$ & & $1(0.50 \%)$ \\
\hline Unknown & 0 & & & $2(1.88 \%)$ & & 0 \\
\hline
\end{tabular}

Type 1-5 advanced type, AN Helicobacter pylori-uninfected group, AP $H$. pylori-infected group, BCD classification of gastric cancer patients, $P P I$ proton pump inhibitors [14]

$n$ lesion numbers

${ }^{\text {a }}$ 0-I, 0-IIa, 0-IIb, 0-IIc; superficial type, tumor confined to the mucosa or submucosa

$(p=0.077)$. The mean tumor size of group AN was the smallest ( $p=0.048$ vs. group AP, $p=0.0068$ vs. group BCD), and group AP had smaller tumor sizes than group $\operatorname{BCD}(p=0.08)$. Concerning the macroscopic features, all patients in group AN showed 0-IIc (flat depressed type) morphology, and the prevalence of 0 -IIc cancer was higher in group AP than in group BCD; however, there were no significant differences. Histologically, all tumors in Group AN showed the undifferentiated type (poorly differentiated adenocarcinoma, 1; signet-ring cell carcinoma, 6) $(p<0.0001)$, whereas the differentiated type was the dominant type in group AP and group BCD. In terms of the histological type, there was no significant difference between groups $\mathrm{AP}$ and $\mathrm{BCD}(p=0.87)$. There was no significant difference in tumor location or depth among the three groups. 


\section{Serum anti-H. pylori antibody titer in each group}

All patients in group A (AN and AP) were negative for the anti-H. pylori antibody titer test $(<10 \mathrm{U} / \mathrm{ml})$. As demonstrated in Table 2, $6(85.7 \%)$ of 7 patients in group AN showed titers below the sensitivity limit $(<3 \mathrm{U} / \mathrm{ml})$. However, $52(51.0 \%)$ of 102 patients in group AP showed negative-high titers, between 3 and $9.9 \mathrm{U} / \mathrm{ml}$. The prevalence of patients with negative-high titers of the anti-H. pylori antibody in group AP was greater than that in group AN; however, it was not statistically significant $(p=0.33)$.

\section{Endoscopic evaluation of corpus atrophy}

All 7 patients in group AN and 74 patients in group AP were subjected to endoscopic assessments of corpus atrophy based on the Kimura and Takemoto classification [13]. As shown in Table 3, the 7 patients in group AN showed $\mathrm{C} 0 / \mathrm{C} 1$, indicating no atrophic change in the gastric corpus. The prevalence of atrophic gastritis was higher in group AP than in group $\mathrm{BCD}(p=0.025)$. In group $\mathrm{AP}, \mathrm{C} 2 / \mathrm{C} 3$ was recognized in 14 patients $(19 \%)$ and open type (O1-O3) was observed in $60(81 \%)$ of 74 patients. In group BCD, $\mathrm{C} 2 / \mathrm{C} 3$ was recognized in 16 patients $(8.9 \%)$ and open type (O1-O3) was observed in $162(91.1 \%)$ of 178.

\section{Serum levels of PG in each group}

We evaluated the serum PG levels in each group. As shown in Table 4, the median PG I levels in group AN, group AP, and group BCD were 71.6, 40.25, and $39.0 \mathrm{ng} / \mathrm{ml}$, respectively. $\mathrm{PG} I$ levels in group $\mathrm{AN}$ were higher than those in groups AP and BCD; however, they were not significantly different ( $p=0.0577,0.0592)$. PG II levels in group AP (median, $8.35 \mathrm{ng} / \mathrm{ml}$ ) were statistically lower than those in group BCD (median, $17.8 \mathrm{ng} / \mathrm{ml}$ ) $(p<0.0001)$. PG I/II ratios in group AN (median, 5.9) were statistically higher than those in group AP (median, 4.41) and in groups BCD (median, 2.1) ( $p=0.0015$, $p<0.0001)$. The PG I/II ratios in group AP were statistically higher than those in group $\mathrm{BCD}(p<0.0001)$.

\section{Discussion}

In Japan, as prevention against gastric cancer death, a mass screening program using radiographic or endoscopic examination has been conducted. $H$. pylori infection and CAG are well-known risk factors for gastric cancer. $H$. pylori infection rates have decreased recently in Japan; the identification of individuals at a high risk for gastric cancer enables establishing efficient prevention strategies.

Table 2 Anti-Helicobacter pylori antibody titer

\begin{tabular}{lllllll}
\hline $\begin{array}{l}\text { Anti- } H . \text { pylori antibody titer } \\
(\mathrm{U} / \mathrm{ml})\end{array}$ & $\begin{array}{l}\text { Group AN } \\
(n=7)\end{array}$ & $\begin{array}{l}p \text { value vs. } \\
\text { AP }\end{array}$ & $\begin{array}{l}p \text { value vs. } \\
\text { BCD }\end{array}$ & $\begin{array}{l}\text { Group AP } \\
(n=102)\end{array}$ & $\begin{array}{l}p \text { value vs. } \\
\text { BCD }\end{array}$ & $\begin{array}{l}\text { Group BCD } \\
(n=183)\end{array}$ \\
\hline$<3$ & $6(85.7 \%)$ & 0.33 & $<0.0001$ & $50(49.0 \%)$ & $<0.0001$ & $4(2.0 \%)$ \\
$3 \leq,<10$ & $1(14.3 \%)$ & & & $52(51.0 \%)$ & $6(3.0 \%)$ \\
$10 \leq$ & 0 & & 0 & $173(94.5 \%)$ \\
\hline
\end{tabular}

$n$ lesion numbers

Table 3 Endoscopic evaluation of corpus atrophy

\begin{tabular}{|c|c|c|c|c|c|c|}
\hline & Group AN $(n=7)$ & $p$ value vs. AP & $p$ value vs. BCD & Group AP $(n=74)$ & $p$ value vs. $\mathrm{BCD}$ & Group BCD $(n=178)$ \\
\hline \multicolumn{7}{|c|}{ Closed type } \\
\hline $\mathrm{C} 0$ & $6(85.71 \%)$ & $<0.0001$ & $<0.0001$ & 0 & 0.0255 & 0 \\
\hline $\mathrm{C} 1$ & $1(14.29 \%)$ & & & 0 & & 0 \\
\hline $\mathrm{C} 2$ & 0 & & & $4(5.41 \%)$ & & $1(0.56 \%)$ \\
\hline $\mathrm{C} 3$ & 0 & & & $10(13.51 \%)$ & & $15(8.43 \%)$ \\
\hline \multicolumn{7}{|c|}{ Open type } \\
\hline $\mathrm{O} 1$ & 0 & & & $16(21.62 \%)$ & & $46(25.84 \%)$ \\
\hline $\mathrm{O} 2$ & 0 & & & $30(40.54 \%)$ & & $94(52.81 \%)$ \\
\hline $\mathrm{O} 3$ & 0 & & & $14(18.92 \%)$ & & $22(12.36 \%)$ \\
\hline
\end{tabular}

$n$ lesion numbers 
Table 4 Serum levels of pepsinogen (PG)

\begin{tabular}{|c|c|c|c|c|c|c|}
\hline & Group AN $(n=7)$ & $p$ value vs. AP & $p$ value vs. $\mathrm{BCD}$ & Group AP $(n=102)$ & $p$ value vs. BCD & Group BCD $(n=183)$ \\
\hline PG I (ng/ml) & & 0.0577 & 0.0592 & & 0.2236 & \\
\hline Average & 123.3 & & & 51.8 & & 46.4 \\
\hline SD & 138.1 & & & 44.2 & & 41.0 \\
\hline Median & 71.6 & & & 40.25 & & 39.0 \\
\hline PG II (ng/ml) & & 0.4246 & 0.6625 & & $<0.0001$ & \\
\hline Average & 19.6 & & & 11.6 & & 22.1 \\
\hline SD & 20.14 & & & 9.5 & & 20.7 \\
\hline Median & 12.8 & & & 8.35 & & 17.8 \\
\hline PG I/II ratio & & 0.0155 & $<0.0001$ & & $<0.0001$ & \\
\hline Average & 6.20 & & & 5.03 & & 2.27 \\
\hline SD & 0.80 & & & 3.17 & & 1.32 \\
\hline Median & 5.90 & & & 4.41 & & 2.10 \\
\hline
\end{tabular}

$n$ lesion numbers

The serological risk prediction system combining the PG test and anti- $H$. pylori antibody testing is widely available to large populations. In this system, subjects are classified into four groups (A, B, C, D), and CAG as a high-risk group for gastric cancer is diagnosed using the PG test and anti-H. pylori antibody serology. In particular, group A patients (H. pylori negative and PG negative) should have an $H$. pylori-uninfected status.

It seems plausible that the $\mathrm{ABC}$ classification system in combination with radiographic or endoscopic examinations would contribute to the establishment of a more efficient screening program for gastric cancer by concentrating on the high-risk population. However, the ABC classification system has still unfortunately lacked sufficient evidence to reduce mortality from gastric cancer. Therefore, the Japanese guidelines for gastric cancer screening in 2014 have not yet recommended the introduction of the $\mathrm{ABC}$ classification system into the population-based screening program [15]. In these guidelines, nevertheless, endoscopic examinations as well as a radiographic screening are newly recommended as elements of population-based screening. In the near future in Japan, endoscopic screening for gastric cancer would increase in a population-based program. The $A B C$ classification system is important for the effective selection of subjects to undergo endoscopic screening. However, the diagnostic accuracy used to discriminate the H. pylori-uninfected population that is truly at low risk is a limitation of the screening process.

Group A subjects are regarded as $H$. pylori uninfected and as having a low risk for gastric cancer development. Therefore, it is likely that these subjects may be excluded from the gastric cancer mass screening system [16]. Indeed, in our study, we found that only seven patients with gastric cancer were subclassified into group AN. As demonstrated in previous reports, gastric cancer patients in group AN were younger than those in other groups, and the histological features of their gastric cancer were predominantly the undifferentiated type [11]. Recently, fundic gland-type cancer was reported to be a representative feature of $H$. pylori-uninfected gastric cancer [17]. However, fundic gland-type cancer was not included in our recent study, suggesting this is not a major part of $H$. pyloriuninfected gastric cancer. Concerning endoscopic or serum markers, none of the seven patients showed endoscopic atrophy in the corpus, and six of seven were below the measurement sensitivity $(<3.0)$ in anti-H. pylori antibody titers.

On the other hand, Boda et al. reported that more than $10 \%$ of gastric cancer patients were classified into group A, suggesting that some patients with gastric cancer risk may be included in group A [12]. Recently, Itoh et al. also reported the aberration of high-risk subjects in group A by using radiologic findings [18]. In the present study, in the multicenter study we conducted in Japan, we clarified that (1) some gastric cancer patients were found in group A and (2) that only approximately $7 \%$ of all gastric cancer patients in group A were regarded as $H$. pylori uninfected. These findings suggest that some high-risk patients for gastric cancer may be included in group A, even though testing negative for $\mathrm{H}$. pylori and $\mathrm{CAG}$, and this is a crucial issue for applying the $\mathrm{ABC}$ system for a cancer mass screening program. False-negative results of anti- $H$. pylori antibody or PG tests may provide a reason for this discrepancy. It is well known that PG levels are influenced by use of PPIs [19]. In spite of careful by interviewing, some PPI users may have been included in this study. Serum PG levels will increase with PPI use or renal failure, which must not be neglected in the medical interview.

As already mentioned, the majority of group A patients with gastric cancer were classified into group AP. The 
clinicopathological features of group AP were as follows: compared with group AN, they were older, had predominantly differentiated-type cancer, endoscopically visualized CAG, and a lower PG I/II ratio. Also, as compared with the BCD group, the depressed-type tumor was dominant, PG II levels were lower, and PGI/II ratios were higher. Group AP had CAG and mild inflammation without anti-H. pylori antibody. These results strongly suggest that these characteristics resemble those typical of patients after successful eradication therapy of $H$. pylori. Group AP subjects might already have unrecognized $H$. pylori eradication.

In subjects who have previously undergone eradication therapy, anti-H. pylori antibody and PG tests show negative results; therefore, these must be excluded from the serological predictive test. To decrease the proportion of patients in group AP, a medical interview discussing eradication is important. However, patients with natural or accidental $H$. pylori eradication (by antibiotics used for infectious diseases other than $H$. pylori infection) may not be recognized using a standard interview. The prevalence of accidentally eradicated patients among healthy Japanese subjects is unknown, but we should recognize that there may be a certain number of such patients. To identify these subjects, endoscopic or radiologic evaluation of their gastric mucosal status might be helpful.

The precise diagnosis of patients in group AN $(H$. pylori-uninfected group) is necessary to reduce the falsenegative evaluation of gastric cancer risk. A previous report suggested that the subjects whose anti- $H$. pylori antibody titers were between 3 and 9.9 should be treated as having a high risk [18]. In addition, these subjects should receive gastrointestinal endoscopic examinations. Also, in the PG test, an advanced cutoff level [PGII $\geq 10$ or PG I/II ratio $\leq 5$ ] was reported to improve the accurate diagnosis of $\mathrm{H}$. pylori-induced CAG [20].

There are some limitations to this study. The use of PPIs may be the most likely concern. Another problem is the determination of $H$. pylori status in our subjects. For the precise determination of $H$. pylori status, more than two tests should be carried out in each subject. In this study, we mainly used the serum anti- $H$. pylori antibody titer test and the endoscopic findings.

When we introduce the $\mathrm{ABC}$ classification system for gastric cancer mass screening, all should be aware of the presence of gastric cancer patients in group A who are wrongly judged as having no risk for gastric cancer development. In group A patients with gastric cancer, only $7 \%$ of patients were $H$. pylori uninfected. This is a crucial problem in the current $\mathrm{ABC}$ classification system, and a revised method should be proposed as soon as possible.

\section{Compliance with ethical standards}

Conflict of interest No author has conflicts of interest or financial arrangements that could potentially influence the described research.

Competing interests The authors have no competing interests.

Human rights statement and informed consent All procedures followed were in accordance with the ethical standards of the responsible committee on human experimentation (institutional and national) and with the Declaration of Helsinki of 1964 and later versions. Informed consent or a substitute for it was obtained from all patients before they were included in the study.

\section{References}

1. Ministry of Health, Labour and Welfare: vital statistics Japan 2012.

2. International Agency for Research on Cancer working group on the evaluation of carcinogenic risks to humans (1994) International Agency for Research on Cancer, Lyon, France, pp 177-240.

3. Correa P. Helicobacter pylori and gastric carcinogenesis. Am J Surg Pathol. 1995;19:S37-43.

4. Uemura N, Okamoto S, Yamamoto S, Yamamoto S, Matsumura $\mathrm{N}$, Yamaguchi S, Yamakido $\mathrm{M}$, et al. Helicobacter pylori infection and the development of gastric cancer. N Engl J Med. 2001;345:784-9.

5. Miki K, Ichinose M, Shimizu A, Huang SC, Oka H, Furihata C, Matsushima T, Takahashi K, et al. Serum pepsinogens as a screening test of extensive chronic gastritis. Gastroenterol Jpn. 1987;22(2):133-41.

6. Kiyohira K, Yoshihara M, Ito M, Haruma K, Tanaka S, Chayama K. Serum pepsinogen concentration as a marker of Helicobacter pylori infection and the histologic grade of gastritis: evaluation of gastric mucosa by serum pepsinogen levels. J Gastroenterol. 2003;38(4):332-8.

7. Miki K. Gastric cancer screening using the serum pepsinogen test method. Gastric Cancer. 2006;9:245-53.

8. Miki K. Gastric cancer screening by combined assay for serum anti-Helicobacter pylori IgG antibody and serum pepsinogen levels: "ABC method". Proc Jpn Acad Ser B Phys Biol Sci. 2011;87(7):405-14.

9. Inoue K, Fujisawa T, Haruma K. Assessment of degree of health of the stomach by concomitant measurement of serum pepsinogen and serum Helicobacter pylori antibodies. Int J Biol Marker. 2010;25(4):207-12.

10. Terasawa T, Nishida H, Kato K, Miyashiro I, Yoshikawa T, Takaku R, et al. Prediction of gastric cancer development by serum pepsinogen test and Helicobacter pylori seropositivity in eastern Asians: a systematic review and meta-analysis. PLoS One. 2014;9(10):e109783.

11. Matsuo T, Ito M, Takata S, Tanaka S, Yoshihara M, Chayama K. Low prevalence of Helicobacter pylori-negative gastric cancer among Japanese. Helicobacter. 2011;16:415-9.

12. Boda T, Ito M, Yoshihara M, Kitamura Y, Matsuo T, Oka S, et al. Advanced method for evaluation of gastric cancer risk by serum markers: determination of true low-risk subjects for gastric neoplasm. Helicobacter. 2014;19:1-8.

13. Kimura K, Takemoto T. An endoscopic recognition of the atrophic border and its significance in chronic gastritis. Endoscopy. 1969;20:1161-81. 
14. Japanese Gastric Cancer Association. Japanese classification of gastric carcinoma (3rd English edition). Gastric Cancer. 2011;14:101-12.

15. Research Center For Cancer Prevention And Screening. National Cancer Center: Japanese guidelines for gastric cancer screening. 2014. http://canscreen.ncc.go.jp/pdf/iganguide2014_150421.pdf.

16. Asaka M, Kato M, Sakamoto N. Roadmap to eliminate gastric cancer with Helicobacter pylori eradication and consecutive surveillance in Japan. J Gastroenterol. 2014;49(1):1-8.

17. Ueyama H, Yao T, Nakashima Y, Hirakawa K, Oshiro Y, Hirahashi $\mathrm{M}$, et al. Gastric adenocarcinoma of fundic gland type (chief cell predominant type): proposal for a new entity of gastric adenocarcinoma. Am J Surg Pathol. 2010;34(5):609-19.
18. Itoh T, Saito M, Marugami N, Hirai T, Marugami A, Takahama J, et al. Correlation between the $\mathrm{ABC}$ classification and radiological findings for assessing gastric cancer risk. Jpn J Radiol. 2015;33(10):636-44.

19. Agréus L, Storskrubb T, Aro P, Ronkainen J, Talley NJ, Sipponen P. Clinical use of proton-pump inhibitors but not H2-blockers or antacid/alginates raises the serum levels of amidated gastrin-17, pepsinogen I and pepsinogen II in a random adult population. Scand J Gastroenterol. 2009;44(5):564-70.

20. Kitamura Y, Yoshihara M, Ito M, Boda T, Matsuo T, Kotachi T, et al. Diagnosis of Helicobacter pylori-induced gastritis by serum pepsinogen levels. J Gastroenterol Hepatol. 2015;30(10):1473-7. 